Opinion

\title{
Covid-19, Carnosine and Cognition
}

\author{
Alan R Hipkiss* \\ Aston Research Centre for Healthy Ageing (ARCHA), Aston University, UK
}

\begin{abstract}
The possible ameliorative roles of the dipeptide carnosine with respect to Covid-19 viral infection and associated pathologies are discussed. In particular carnosine's ability to suppress age-related changes in carbohydrate metabolism which normally exacerbate Covid-19-induced dysfunction as well as the dipeptide's anti-inflammatory activity is considered. As carnosine is normally present in the olfactory lobe and that anosmia (loss of sense of smell) is a common feature of Covid-19's effect on humans, the possibility that nasal administration of carnosine could be therapeutic is considered as a means of raising levels of the dipeptide in the olfactory lobe and thereby alleviates virus-mediated neuropathology.
\end{abstract}

Keywords: Carnosine, Glycolysis, Glycation, Diabetes, Senescence, Reprogramming, Brain

\section{Carbohydrate Metabolism, Covid-19 and Aging}

There is substantial evidence which indicates a close association between Covid-19, type-2 diabetes and much age-associated dysfunction mediated by enhanced carbohydrate catabolism i.e. glycolysis. It appears that either (i) elevated glucose metabolism enhances viral infection and replication, ${ }^{1-3}$ or (ii) Covid-19 exerts a causal role towards diabetes and related phenomena. ${ }^{4}$ The close association between diabetes and Covid-19 is further illustrated by the finding that enhanced non-enzymic protein glycosylation (glycation) accompanies both phenomena. ${ }^{5-7}$ Much non-enzymic protein glycation is mediated by the glycolytic intermediates, the triose-phosphates dihydroxyacetone phosphate and glyceraldehyde-3-phosphate and their highly reactive decomposition product, methylglyoxal (MG). Indeed, MG is regarded as responsible for much post-synthetic protein, lipid and nucleic acid glycation which accompanies age-related dysfunction. ${ }^{8}$

Studies show that Covid-19 virus infection induces an up regulation in glycolytic flux, presumably in order to produce the necessary nucleic acid and protein precursors (nucleotides and amino acids) for viral replication. ${ }^{9}$ It is also relevant to note that enhanced carbohydrate catabolism is frequently found to accelerate the onset of much age-related dysfunction, whereas partial suppression of glycolysis delays aging onset. ${ }^{10}$ Aging is a risk factor for Covid-19 induced mortality; ${ }^{11}$ not only does patient age constitute a risk factor for virus-induced mortality, but this is also reflected at the cellular level; a recent study showed that clearance of senescent cells decreased the severity of Covid-19 associated pathology. ${ }^{12}$ The naturally-occurring dipeptide carnosine (beta-alanyl-L-histidine) was recently shown to facilitate the phagocytic elimination of senescent cells ${ }^{13}$ in a model system, and clearance of senescent cells, as noted above, can decrease the severity of Covid-19 -associated pathology. ${ }^{12}$

\section{Other Properties of Carnosine Which May Help to Sup- press Covid-19 Pathology}

A particular feature of Covid-19 is a phenomenon called a cytokine storm, which is a pro-inflammatory condition mediated systemically by leukocytes of the immune system ${ }^{14}$ and/or macrophages, ${ }^{1}$ following infection..$^{15}$ Macrophages exist in two forms,
Quick Response Code:

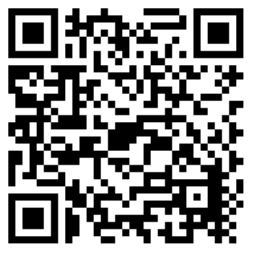

*Corresponding author: Alan R Hipkiss, Aston Research Centre for Healthy Ageing (ARCHA), Aston University, Birmingham, B4 7ET, UK, and Sport, Health and Performance Enhancement (SHAPE) Research Centre, Nottingham Trent University, Nottingham, NG11 8NS, UK

Received: 27 May, 2021

Published: 08 July, 2021

Citation: Hipkiss AR. Covid-19, Carnosine and Cognition. SOJ Neuro Neurosci. 2021;1(2):1-4. DOI: 10.53902/SOJNN.2021.01.000506 
M1 and M2. The M1phenotype is the pro-inflammatory state which releases pro-inflammatory cytokines, whereas the M2 phenotype releases anti-inflammatory agents. Furthermore, the M1 and M2 phenotypes differ in terms of their energy metabolism, while the M1 macrophage is highly glycolytic, the M2 form is more aerobic. Macrophage metabolic reprogramming is a recognised feature of control of inflammation. ${ }^{6}$

Carnosine has been shown to reprogram macrophage energy metabolism from their pro-inflammatory M1 state into the anti-inflammatory M2 state, ${ }^{16}$ thereby suppressing secretion of pro-inflammatory cytokines IL-1beta and IL-6, and upregulating expression of the anti-inflammatory cytokine TGF-beta- 1 and the protective factor NRF-2. These changes are most probably mediated via changes in mRNA translation, by the dipeptide suppressing of cap-dependent mRNA translation, and enhancing cap-independent mRNA translation. ${ }^{17}$ interestingly, cap-independent mRNA translation has been reported to be up regulated in long-lived mice ${ }^{18}$ which results in enhanced mitochondrial biogenesis, including the mitochondrial Lon-1 peptidase and the protective peptide humanin. ${ }^{19}$

It is interesting to note that the Covid-19 virus (SARS-CoV-2) also uses glutamine as a metabolic source of macromolecule precursors, in addition to glucose. ${ }^{20}$ Importantly, carnosine can inhibit glutamine metabolism, at least in human glioma cells, by accelerating the proteolysis of glutamine synthetase, ${ }^{21}$ which could further decrease the synthesis of macromolecular precursors (amino acids and purine and pyrimidine nucleotides) in addition to carnosine's suppressive effects on glycolysis. However, whether carnosine is inhibitory to glutamine metabolism generally (i.e. other cell types) remains to be studied.

Carnosine is a dipeptide consisting of beta-alanine and histidine and is normally found mostly in skeletal muscle and in the olfactory bulb in the brain..$^{22}$ Carnosine has been described as enigmatic, ${ }^{23}$ and it is clearly pluripotent in its activities. Following its discovery over 100 years ago, ${ }^{24}$ many properties have been ascribed to carnosine; at a biochemical level these include hydrogen ion buffer, antioxidant, anti-glycating agent and aldehyde-scavenger. Physiologically, effects on muscle strength are claimed, ${ }^{25}$ as are anti-inflammatory effects ${ }^{26}$ and anti-ageing activities. ${ }^{27}$

The dipeptide has also been shown to inhibit growth of transformed cells. ${ }^{28}$ Metabolic effects such as partial inhibition of glycolysis, ${ }^{29}$ and enhancement of mitochondrial activity ${ }^{30}$ and proteolysis $^{31}$ have also been detected. Carnosine has also been shown to delay senescence in cultured human fibroblasts and to rejuvenate senescent cells. ${ }^{28}$ Beneficial effects towards senescence-accelerated mice ${ }^{32}$ have also been described, as well as suppression of diabetes and protein glycation in mice. ${ }^{33}$ Carnosine also facilitates macrophage-mediated clearance of senescent cells. ${ }^{34}$

It is likely that carnosine's effects are multifactorial, not only can the dipeptide affect metabolism, but it has recently been shown that prolonged fasting causes blood levels of carnosine (presumably intra-erythrocytic) to increase ${ }^{35}$ along with a number of other anti-oxidants. A recent study has produced evidence suggesting that carnosine may act as an inhibitor of angiotensin-converting enzyme 2 (ACE2) which could then inhibit viral cellular entry via this protein. ${ }^{36}$ Thus carnosine could suppress not only viral infection but also Covid-19-induced metabolic changes.

\section{Carnosine, Human Aging and Cognition}

A recent study has revealed that Covid-19-related anosmia is accompanied by viral infection of the olfactory neuro-epithelium and the olfactory bulb, which may provide viral entry to the rest of the brain..$^{37}$ and impact brain function generally. ${ }^{38}$ Carnosine is known to be present not only in human muscle but also in the olfactory bulb. More recently the dipeptide has been detected in human erythrocytes, in amounts which decline with the age of the human source. ${ }^{39}$ Furthermore, blood levels of carnosine have been reported to be very low in patients suffering from age-related macular degeneration, ${ }^{40}$ whilst very low serum levels of $\mathrm{N}$-acetyl-carnosine (i.e. resistant to serum carnosinase) are strongly associated with frailty in humans. ${ }^{41}$

There have been a number of studies on the possible effects of dietary carnosine supplementation in humans ${ }^{34,42}$ but the presence of serum carnosinase which ensures the rapid destruction of the dipeptide, limits the impact of dietary-supplied dipeptide. It is likely that the majority of carnosine in blood is due to its presence within erythrocytes; presumably the dipeptide is synthesized during erythropoiesis. As erythrocytes are normally replaced after a lifetime of around 120 days in humans, it can be assumed that any study hoping to detect changes in blood (erythrocyte) carnosine levels should last for at least 3-4 months. One speculates whether erythrocytes deliver carnosine to the tissues in addition to oxygen. It is also possible that carnosine might somehow facilitate carbon dioxide uptake from the tissues and its release in the lungs as the dipeptide has been reported to affect the activity of carbonic anhydrase. ${ }^{43}$ It is never-the-less interesting that carnosine supplementation has revealed a beneficial effect towards schizophrenia ${ }^{44,45}$ and increased protein glycation in erythrocytes from schizophrenics has been reported. ${ }^{46,47}$

Furthermore, childhood trauma has been reported to increase methylation of the carnosine synthase gene thereby decreasing carnosine synthesis which may contribute to the accelerated biological aging upon becoming adults. ${ }^{48}$ There are no reports on whether changes in carnosine synthesis accompany psychological trauma in adults, but it is known that carnosine can exert beneficial effects following physical injury ${ }^{49,50}$ and there are also reports of positive effects of carnosine towards glycaemic control and obesity. ${ }^{51}$ Animal studies indicate that the dipeptide may suppress anxiety. ${ }^{52}$ The dipeptide's ability to clear senescent cells ${ }^{33}$ may alleviate brain inflammation and age-related cognitive impairment. ${ }^{53}$ Furthermore, carnosine-mediated inhibition of advanced glycation end-product (AGE) formation in mice could also suppress age-related cognitive dysfunction. ${ }^{54}$ These observations suggest that carnosine's therapeutic potential should be explored in humans. While effects of Covid-19 on neurological activity have been discussed ${ }^{55-57}$ and deleterious changes in the CNS detected such as inflammation have 
been described, ${ }^{58}$ it remains uncertain whether these are specific to Covid-19, or just a response to general infection. Nevertheless there is evidence showing that the presence of AGEs is a risk factor for Covid-19-associated mortality. ${ }^{59}$

\section{Conclusion}

It is possible that carnosine could exert protective activity towards Covid-19 infection and subsequent pathology. ${ }^{60,61}$ Not only does the dipeptide suppress some of the metabolic changes consequent upon viral infection, it may actually inhibit viral entry too. ${ }^{36}$ Given that the virus enters the lungs, it is possible that raising the olfactory level of carnosine via nasal administration could be an effective strategy first to inhibit viral entry and then raise carnosine levels in the CNS generally, which may protect against glycation-induced dysfunction. Nasal delivery of carnosine ${ }^{31,62}$ via a douche of carnosine in solution is an obvious possibility, however one might also consider a carnosine powder, akin to snuff as used years ago; indeed "snorting" carnosine would be medically preferable to the so-called "recreational" powders which apparently do reach the brain. ${ }^{63-65}$

\section{Acknowledgement}

None.

\section{Funding}

None.

\section{Con flict of Interest}

Author declares that there is no conflict of interest.

\section{References}

1. Codo AC, Davanzo GG, Monteiro LB, et al. Elevated Glucose Levels Favor SARS-CoV-2 Infection and Monocyte Response through a HIF-1 $\alpha$ / Glycolysis-Dependent Axis. Cell Metab. 2020;32(3):437-446.

2. Shah H, Khan MSH, Dhurandhar NV. The triumvirate: why hypertension, obesity, and diabetes are risk factors for adverse effects in patients with COVID-19. Acta Diabetol. 2021;58(7):831-843.

3. Klonoff DC, Messler JC, Umpierrez GE, et al. Association Between Achieving Inpatient Glycemic Control and Clinical Outcomes in Hospitalized Patients With COVID-19: A Multicenter, Retrospective Hospital-Based Analysis. Diabetes Care. 2021;44(2):578-585.

4. Accili D. Can COVID-19 cause diabetes? Nat Metab. 2021;3(2):123125.

5. Chiappalupi S, Salvadori L, Vukasinovic A, et al. Targeting RAGE to prevent SARS-CoV-2-mediated multiple organ failure: Hypotheses and perspectives. Life Sci. 2021;272:119251.

6. Guo C, Li B, Ma H, et al. Single-cell analysis of two severe COVID-19 patients reveals a monocyte-associated and tocilizumab-responding cytokine storm. Nat Commun. 2020;11(1):3924.

7. Sartore G, Ragazzi E, Faccin L, et al. A role of glycation and methylation for SARS-CoV-2 infection in diabetes? Med Hypotheses. 2020;144:110247.

8. Thornalley PJ. Protein and nucleotide damage by glyoxal and methylglyoxal in physiological systems--role in ageing and disease. Drug Metabol Drug Interact. 2008;(1-2):125-150.

9. Ajaz S, McPhail MJ, Singh KK. Mitochondrial metabolic manipulation by SARS-CoV-2 in peripheral blood mononuclear cells of patients with COVID-19. Am J Physiol Cell Physiol. 2021;320(1):C57-C65.

10. Ingram DK, Roth GS. Glycolytic inhibition as a strategy for developing calorie restriction mimetics. Exp Gerontol. 2011;46(2-3):148-154.

11. Pang L, Liu Y, Shen M, et al. Influence of aging on deterioration of patients with COVID-19. Aging. 2020;12(24):26248-26262.

12. Nehme J, Borghesan M, Mackedenski S, et al. Cellular senescence as a potential mediator of COVID-19 severity in the elderly. Aging Cell. 2020;19(10):e13237.

13. Li X, Yang K, Gao S, et al. Carnosine Stimulates Macrophage-Mediated Clearance of Senescent Skin Cells Through Activation of the AKT2 Signaling Pathway by CD36 and RAGE. Front. Pharmacol. 2020.

14. Liu XQ Jiang L, Lei L, et al. Carnosine alleviates diabetic nephropathy by targeting GNMT, a key enzyme mediating renal inflammation and fibrosis. Clin Sci (Lond). 2020;134(23):3175-3193.

15. Delattre H, Sasidharan K, Soyer OS. Inhibiting the reproduction of SARS-CoV-2 through perturbations in human lung cell metabolic network. Life Sci Alliance. 2020;4(1):e202000869.

16. Fresta CG, Fidilio A, Lazzarino G, et al. Modulation of Pro-Oxidant and Pro-Inflammatory Activities of M1 Macrophages by the Natural Dipeptide Carnosine. Int J Mol Sci. 2020;21(3):776.

17. Son DO, Satsu H, Kiso Y, et al. Inhibitory effect of carnosine on interleukin-8 production in intestinal epithelial cells through translational regulation. Cytokine. 2008;42(2):265-276.

18. Ozkurede U, Kala R, Johnson C, et al. Cap-independent mRNA translation is up regulated in long-lived endocrine mutant mice. J Mol Endocrinol. 2019;63(2):123-138.

19. Yen K, Mehta HH, Kim SJ, et al. The mitochondrial derived peptide humanin is a regulator of lifespan and health-span. Aging (Albany NY). 2020;12(12):11185-11199.

20. Bharadwaj S, Singh M, Kirtipal N, et al. SARS-CoV-2 and Glutamine: SARS-CoV-2 Triggered Pathogenesis via Metabolic Reprograming of Glutamine in Host Cells. Front Mol Bioscience. 2021;7:627842.

21. Fang YJ, Wu M, Chen HN, et al. Carnosine suppresses human glioma cells under normoxic and hypoxic conditions partly via inhibiting glutamine metabolism. Acta Pharmacol Sin. 2021;42(5):767-779.

22. Colin Barenque L, Bizarro Nevares P, Gonzalez Villalva A, et al. Neuroprotective effect of carnosine in the olfactory bulb after vanadium inhalation in a mouse model. Int J Exp Pathol. 2018;99(4):180-188.

23. Bauer K. Carnosine and homocarnosine, the forgotten, enigmatic peptides of the brain. Neurochem Res. 2005;30(10):1339-1345.

24. Boldyrev AA, Aldini G, Derave W. Physiology and pathophysiology of carnosine. Physiol Rev. 2013;93(4):1803-1845.

25. Bassinello D, de Salles Painelli V, Dolan E, et al. Beta-alanine supplementation improves isometric, but not isotonic or isokinetic strength endurance in recreationally strength-trained young men. Amino Acids. 2019;51(1):27-37.

26. Aldini G, de Courten B, Regazzoni L, et al. Understanding the antioxidant and carbonyl sequestering activity of carnosine: direct and indirect mechanisms. Free Radic Res. 2020;11:1-10.

27. Hipkiss AR, Baye E, de Courten B. Carnosine and the processes of ageing. Maturitas. 2016;93:28-33.

28. Holliday R, McFarland GA. Inhibition of the growth of transformed and neoplastic cells by the dipeptide carnosine. $\mathrm{Br} J$ Cancer. 1996;73(8):966-971.

29. Cartwright SP, Bill RM, Hipkiss AR. L-carnosine affects the growth of Saccharomyces cerevisiae in a metabolism-dependent manner. PLoS One. 2012;7(9):e45006. 
30. Ghanbarinejad V, Ahmadi A, Niknahad H, et al. Carnosine Mitigates Manganese Mitotoxicity in an In Vitro Model of Isolated Brain Mitochondria. Adv Pharm Bull. 2019;9(2):294-301.

31. Hipkiss AR. Could carnosine suppress zinc-mediated proteasome inhibition and neurodegeneration? Therapeutic potential of a non-toxic but non-patentable dipeptide. Biogerontology. 2005;6(2):147-149.

32. Boldyrev AA, Yuneva MO, Sorokina EV, et al. Antioxidant systems in tissues of senescence accelerated mice. Biochemistry (Mosc). 2001;66(10):1157-1163.

33. Zhang R, Wang X, Ni L, et al. COVID-19: Melatonin as a potential adjuvant treatment. Life Sci. 2020;250:117583.

34. Masuoka N, Lei C, Li H, et al. Influence of Imidazole-Dipeptides on Cognitive Status and Preservation in Elders: A Narrative Review. Nutrients. 2021;13(2):397.

35. Kondoh $\mathrm{H}$, Teruya T, Yanagida M. Metabolomics of human fasting: new insights about old questions. Open Biol. 2020;10(9):200176.

36. Saadah LM, Deiab GIA, Al Balas Q et al. Carnosine to Combat Novel Coronavirus (nCoV): Molecular Docking and Modeling to Cocrystallized Host Angiotensin-Converting Enzyme 2 (ACE2) and Viral Spike Protein. Molecules. 2020;25(23):5605.

37. de Melo GD, Lazarini F, Levallois S, et al. COVID-19-related anosmia is associated with viral persistence and inflammation in human olfactory epithelium and brain infection in hamsters. Sci Transl Med. 2021;3(596):eabf8396.

38. Hasegawa Y, Namkung H, Smith A, et al. Causal impact of local inflammation in the nasal cavity on higher brain function and cognition. Neurosci Res. 2021;28:S0168-0102(21)00093-00096.

39. Chaleckis R, Murakami I, Takada J, et al. Individual variability in human blood metabolites identifies age-related differences. Proc Natl Acad Sci USA. 2016;113(16):4252-4259.

40. Chao de la Barca JM, Rondet Courbis B, Ferre M, et al. A Plasma Metabolomic Profiling of Exudative Age-Related Macular Degeneration Showing Carnosine and Mitochondrial Deficiencies. J Clin Med. 2020;9(3):631.

41. Kameda M, Teruya T, Yanagida M, et al. Frailty markers comprise blood metabolites involved in antioxidation, cognition, and mobility. Proc Natl Acad Sci USA. 2020;117(17):9483-9489.

42. Caruso J, Charles J, Unruh K, et al. Ergogenic effects of beta-alanine and carnosine: proposed future research to quantify their efficacy. Nutrients. 2012;4(7):585-601.

43. Vistoli G, Aldini G, Fumagalli L, et al. Activation Effects of Carnosineand Histidine-Containing Dipeptides on human carbonic Anhydrases: A Comprehensive Study. Int J Mol Sci. 2020;21(5):1761.

44. Chengappa KN, Turkin SR, DeSanti S, et al. A preliminary, randomized, double-blind, placebo-controlled trial of L-carnosine to improve cognition in schizophrenia. Schizophr Res. 2012;142(1-3):145-152.

45. Ghajar A, Khoaie-Ardakani MR, Shahmoradi Z, et al. L-carnosine as an add-on to risperidone for treatment of negative symptoms in patients with stable schizophrenia: A double-blind, randomized placebo-controlled trial. Psychiatry Res. 2018;262:94-101.

46. Ishida YI, Kayama T, Kibune Y, et al. Identification of an argpyrimidine-modified protein in human red blood cells from schizophrenic patients: A possible biomarker for diseases involving carbonyl stress. Biochem Biophys Res Commun. 2017;493(1):573-577.

47. Ohnuma T, Nishimon S, Takeda M, et al. Carbonyl Stress and Microinflammation-Related Molecules as Potential Biomarkers in Schizophrenia. Front Psychiatry. 2018;9:82.
48. Marinova Z, Maercker A, Grunblatt E, et al. A pilot investigation on DNA methylation modifications associated with complex posttraumatic symptoms in elderly traumatized in childhood. BMC Res Notes. 2017;10(1):752.

49. Tanaka KI, Sugizaki T, Kanda Y, et al. Preventive Effects of Carnosine on Lipopolysaccharide-induced Lung Injury. Sci Rep. 2017;7:42813.

50. Ommati MM, Heidari R, Ghanbarinejad V, et al. The neuroprotective properties of carnosine in a mouse model of manganism is mediated via mitochondria regulating and anti-oxidative mechanisms. Nutr Neurosci. 2020;23(9):731-743.

51. Menon K, Marquina C, Liew D, et al. Histidine-containing dipeptides reduce central obesity and improve glycaemic outcomes: A systematic review and meta-analysis of randomized controlled trials. Obes Rev. 2020;21(3):e12975.

52. Hoffman JR, Gepner Y, Cohen H. $\beta$-Alanine supplementation reduces anxiety and increases neurotrophin expression in both young and older rats. Nutr Res. 2019;62:51-63.

53. Ogrodnik M, Evans SA, Fielder E, et al. Whole-body senescent cell clearance alleviates age-related brain inflammation and cognitive impairment in mice. Aging Cell. 2021;20(2):e13296.

54. khter F, Chen D, Akhter A, et al. High Dietary Advanced Glycation End Products Impair Mitochondrial and Cognitive Function. J Alzheimers. 2020;76(1):165-178.

55. Ellul MA, Benjamin L, Singh B, et al. Neurological associations of COVID-19. Lancet Neurol. 2020;19(9):767-783.

56. Mattioli F, Stampatori C, Righetti F, et al. Neurological and cognitive sequelae of Covid-19: a four month follow-up. J Neurol. 1:1-7.

57. Ren Z, Xin Y, Ge J, et al. Psychological Impact of COVID-19 on College Students After School Reopening: A Cross-Sectional Study Based on Machine Learning. Front Psychol. 2021;12:641806.

58. Farhadian SF, Seilhean D, Spudich S. Neuropathogenesis of acute coronavirus disease 2019. Curr Opin Neurol. 2021;34(3):417-422.

59. Rojas A, Lindner C, Gonzalez I, et al. Advanced-glycation end-products axis: A contributor to the risk of severe illness from COVID-19 in diabetes patients. World J Diabetes. 2021;12(5):590-602.

60. Hipkiss AR. COVID-19 and Senotherapeutics: Any Role for the Naturally-occurring Dipeptide Carnosine? Aging Dis. 2020;11(4):737741.

61. Feehan J, de Courten M, Apostolopoulos V, et al. Nutritional Interventions for COVID-19: A Role for Carnosine? Nutrients. 2021;13(5):1463.

62. Hipkiss AR. Glycotoxins: Dietary and Metabolic Origins; Possible Amelioration of Neurotoxicity by Carnosine, with Special Reference to Parkinson's Disease. Neurotox Res. 2018;34(1):164-172.

63. Icard P, Lincet $\mathrm{H}, \mathrm{Wu} \mathrm{Z}$, et al. The key role of Warburg effect in SARS$\mathrm{CoV}-2$ replication and associated inflammatory response. Biochimie. 2021;180:169-177.

64. Koike S, Toriumi K, Kasahara S, et al. Accumulation of Carbonyl Proteins in the Brain of Mouse Model for Methylglyoxal Detoxification Deficits. Antioxidants (Basel). 2021;10(4):574.

65. Wang J, Meng W. COVID-19 and diabetes: the contributions of hyperglycemia. J Mol Cell Biol. 2021;12(12):958-962. 